\title{
Solid-solid phase transition in adenine riboswitch crystals driven by large conformational changes induced by ligand
}

\author{
Jason Stagno', Saminathan Ramikrishnan², William F. Heinz ${ }^{3}$, Valentin Magidson ${ }^{4}$, Xiaobing Zuo ${ }^{5}$, \\ Ping $\mathrm{Yu}^{6}$, Yun-Xing Wang ${ }^{7}$ \\ ${ }^{1}$ National Cancer Institute ${ }^{2}$ Center for Structural Biology, Centre for Cancer Research, National \\ Cancer Institute, ${ }^{3}$ Optical Microscopy and Analysis Laboratory, Cancer Research Technology \\ Program, Frederick National L, ${ }^{4}$ Optical Microscopy and Analysis Laboratory, Cancer Research \\ Technology Program, Frederick National L, ${ }^{5}$ X-ray Science Division, Argonne National Laboratory, \\ ${ }^{6}$ National Cancer Institute, ${ }^{7}$ Structural Biophysics Laboratory, National Cancer Institute \\ Jason.stagno@nih.gov
}

\begin{abstract}
A solid-solid phase transition (SSPT) occurs between distinguishable crystalline forms. SSPTs have been studied extensively in metallic alloys, inorganic salt or small organic molecular crystals, but much less so in biomacromolecular crystals. In particular, SSPTs involving large-scale molecular changes that are important to biological function are largely unexplored, yet may enhance our understanding of conformational space. Here, we report a systematic study of the ligand-induced SSPT in crystals of the adenine riboswitch aptamer RNA (riboA) using a combination of polarized video microscopy (PVM), solution atomic force microscopy (AFM), and timeresolved serial crystallography (TRX). The SSPT, driven by large conformational changes induced by ligand, transforms the crystal lattice from monoclinic (apo), to triclinic (intermediate lattice in a ligand-bound conformation), to orthorhombic (final bound conformational and lattice state). Using crystal structures of each state, we mapped out the changes to the crystal packing interfaces, which define the interplay between molecular conformation and crystal phase, which were corroborated by solution AFM. Using PVM to monitor changes in crystal birefringence, we characterized the kinetics of the SSPT in crystals of different sizes and ligand concentration. Together, these studies illustrate a practical approach for characterizing SSPT in biomacromolecular crystals involving large conformational changes, and provide useful spatiotemporal data for informing time-resolved crystallography experiments.
\end{abstract}

